\title{
High Flow Through a Nasal Cannula and CPAP Effect in a Simulated Infant Model
}

\author{
Teresa A Volsko MHHS RRT FAARC, Kathy Fedor RRT-NPS, \\ Jason Amadei RRT, and Robert L Chatburn MHHS RRT-NPS FAARC
}

\begin{abstract}
BACKGROUND: Limited data are available to describe the CPAP effects that can be expected when using high flow with a traditional nasal cannula. OBJECTIVE: To describe the relationship between the pressure generated at the airway opening and flow through a nasal cannula using a simulated infant model. We hypothesized that positive pressure generated by a standard cannula at flows $>2 \mathrm{~L} / \mathrm{min}$ would be minimal and clinically unimportant. METHODS: Nares were simulated with holes drilled in a plastic fixture. A nares template for CPAP prongs served as a sizing template for the holes. Small, medium, and large nares fixtures were constructed and connected to a lung simulator that simulated spontaneous breathing. Respiratory muscle pressure was simulated by setting a waveform and adjusting the amplitude to deliver a range of tidal volumes $\left(V_{T}\right)$ from $3 \mathrm{~mL}$ to $12 \mathrm{~mL}$. Lung compliance and resistance were set at $0.5 \mathrm{~mL} / \mathrm{cm} \mathrm{H}_{2} \mathrm{O}$ and $125 \mathrm{~cm} \mathrm{H}_{2} \mathrm{O} / \mathrm{L} / \mathrm{s}$, respectively. Nasal cannulas were inserted in the model nares. We assured that the prong occlusion of the nares did not exceed $50 \%$. Cannula flow was adjusted from $2-6 \mathrm{~L} / \mathrm{min}$ in $1-\mathrm{L} / \mathrm{min}$ increments. Data were averaged over 20 breaths. Mean airway pressure and percent change in $V_{T}$ were recorded. RESULTS: The greatest effect on $V_{T}($ mean \pm SD $0.16 \pm 0.10 \mathrm{~mL})$ and pressure change (mean \pm SD $0.7 \pm 0.5 \mathrm{~cm} \mathrm{H}_{2} \mathrm{O}$ ) occurred with the premature cannula. The least effect on pressure (mean \pm SD $0.3 \pm 0.22 \mathrm{~cm} \mathrm{H}_{2} \mathrm{O}$ ) and $\mathrm{V}_{\mathrm{T}}$ change (mean $\pm \mathrm{SD} 0.01 \pm 0.02 \mathrm{~mL}$ ) occurred with the infant cannula. CONCLUSIONS: Clinically important pressures were not generated by high flows with a standard nasal cannula. The differences in spontaneous $\mathbf{V}_{\mathbf{T}}$ across all flows were negligible. Key words: nasal CPAP; high-flow nasal cannula; pediatric; oxygen therapy. [Respir Care 2011;56(12): 1893-1900. ㅇ 2011 Daedalus Enterprises]
\end{abstract}

\section{Introduction}

CPAP is often used in preterm neonates to recruit and maintain lung volume. ${ }^{1}$ During CPAP, baseline airway

\footnotetext{
Ms Volsko is affiliated with the Children's Hospital Medical Center of Akron, Akron, Ohio. Ms Fedor is affiliated with the Pediatric Institute; and $\mathrm{Mr}$ Amadei and Mr Chatburn are affiliated with the Respiratory Institute, The Cleveland Clinic, Cleveland Ohio.
}

Mr Amadei presented a version of this paper at the 55th International Respiratory Congress of the American Association for Respiratory Care, held December 5-8, 2009, in San Antonio, Texas.

Mr Chatburn has disclosed relationships with IngMar, Hamilton, Covidien, and Dräger. The other authors have disclosed no conflicts of interest.

Correspondence: Teresa A Volsko MHHS RRT FAARC, Children's Hospital Medical Center of Akron, One Perkins Square, Akron OH 44308. E-mail: tvolsko@chmca.org.

DOI: $10.4187 /$ respcare. 01204 pressure is maintained at a constant value throughout the entire respiratory cycle, during which time the patient encumbers all of the work of breathing. A plethora of literature is available on the physiological effects of CPAP,

See the Related Editorial on Page 1972

especially with regard to infants and children. Clinical trials in the early 1970s through the mid-1980s substantiated the therapeutic value of CPAP among the neonatal and pediatric population, which included enhancing oxygenation by maintaining and/or improving functional residual volume, ${ }^{2}$ reducing airways resistance, ${ }^{3}$ and treating obstructive apnea. ${ }^{4}$ CPAP has been used clinically for more than 30 years, from the delivery room to the intensive care unit, to treat a host of respiratory conditions. The literature reports the use of CPAP as an alternative to intubation and ventilation for the treatment 


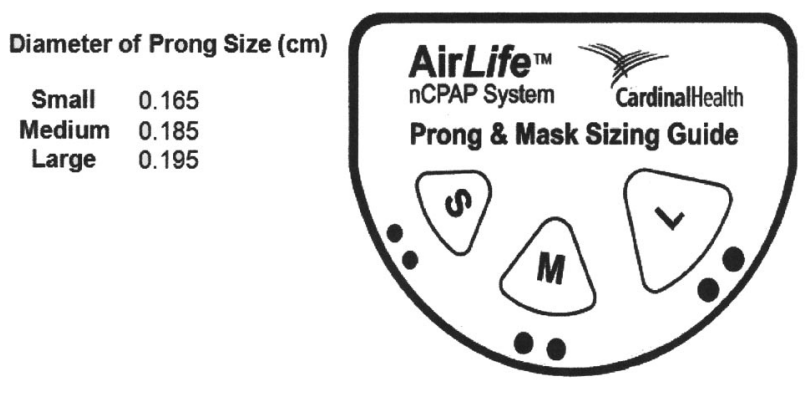

Fig. 1. Template for selecting nasal prong size.

of respiratory distress syndrome, ${ }^{5}$ as a method of preventing extubation failure, ${ }^{6}$ and for management of apnea of prematurity. ${ }^{7}$

CPAP can be delivered by a variety of devices, including bubble CPAP, free-standing infant flow driver, or conventional ventilator. Bubble CPAP provides continuous distending pressure in a relatively simple and inexpensive way. This delivery system directs the infant's exhaled flow as well as the continuous flow of gas provided by the delivery system and through a water reservoir. Bubbles are created as the infant exhales against the resistance provided by the water source. The amount of positive pressure generated during the respiratory cycle is proportional to the depth the circuit directing flow is placed within the reservoir. ${ }^{8}$ Continuous flow generators and conventional ventilators may also be used to deliver CPAP. These devices enhance patient safety with the availability of disconnect, and high-pressure alarms, as well as apnea and tidal volume $\left(\mathrm{V}_{\mathrm{T}}\right)$ alerts when conventional ventilators are used. ${ }^{9}$ Interfaces such as endotracheal tubes, nasal masks, short bi-nasal prongs, or longer single or bi-nasal pharyngeal prongs have been used with premature and term infants. Nasal, oronasal, and/or full-face masks are interfaces that may be used as well with pediatric patients.

The use of a conventional or standard nasal cannula at a high flow rate has also been reported as another method that can be used to deliver CPAP to infants. High-flow oxygen therapy for neonates, defined as continuous flow of greater than $2 \mathrm{~L} / \mathrm{min}$ delivered by nasal cannula, generates continuous positive airway pressure when the cannula flow opposes the infant's spontaneous expiratory flow. ${ }^{7}$ The aforementioned system, in contrast with heated high-flow nasal cannula systems, merely uses a simple bubble humidifier connected to the cannula. Unlike nasal CPAP systems, published data suggest that a tighter seal between the oxygen delivery system and the nose is essential, which presumably results in higher CPAP pressure for a given oxygen flow and ventilatory pattern, and report that the airway pressure generated with high-flow oxygen therapy is variable and unpredictable. ${ }^{7}$ Moreover, the tighter

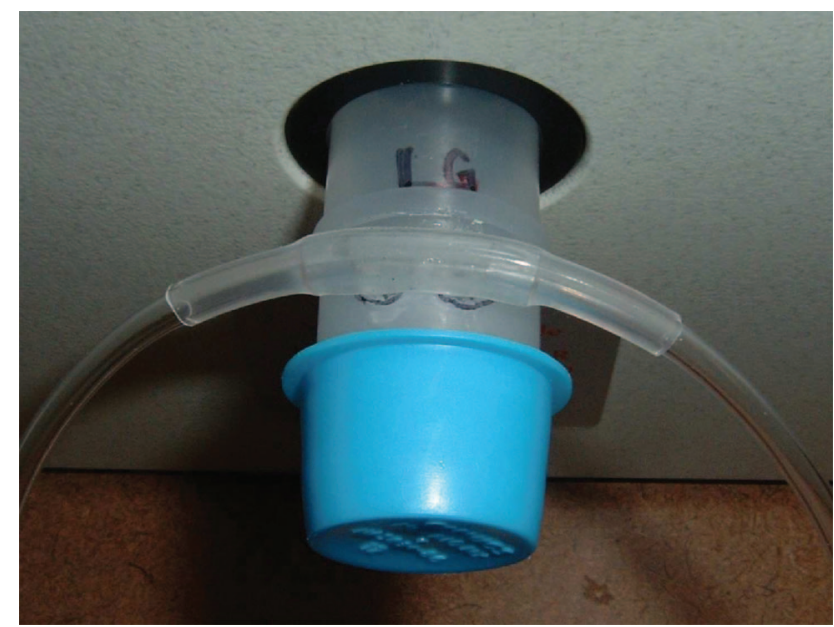

Fig. 2. Model nares, made from a 22-mm inner-diameter adapter, with nasal cannula in place, attached to the lung simulator. The cap on the distal end of the adapter prevents leak, which simulates a closed-mouth scenario. $L G=$ large (ie, the large nasal prongs indicated in Fig. 1).

seal required to obtain therapeutic CPAP would require the nasal cannula to occlude more than $50 \%$ of the nares, which is contrary to standard practice. The use of a tighter seal would proliferate complications such as nasal obstruction, bleeding, and necrosis, which the literature reports are associated with the use of oxygen delivery via nasal cannula. ${ }^{10,11}$

The purpose of this study was to describe the relationship between the pressure generated at the airway opening and the flow through a standard nasal cannula, with a simulated infant lung model. We hypothesized that the positive pressure generated by a correctly sized conventional nasal cannula at flows greater than $2 \mathrm{~L} / \mathrm{min}$ would be minimal and clinically unimportant.

\section{Methods}

\section{Model}

Nares were simulated by drilling holes in a double $22 \mathrm{~mm}$ outer-diameter plastic adapter. The adapter was not modified to replicate the anatomical features of an infant's nasopharynx. The size of the nares were standardized and corresponded with a nares template (AirLife, Cardinal Health, Dublin, Ohio) (Fig. 1). The fixtures were connected to a lung simulator (ASL 5000, with software version 3.1, Ingmar Medical, Pittsburgh, Pennsylvania), which simulated active breathing. The other end of the fixture was capped, to represent a nasopharyngeal model with a closed mouth (Fig. 2). The integrity of the model and its ability to generate CPAP was validated in a published study that evaluated 5 neonatal nasal CPAP systems. ${ }^{12} \mathrm{We}$ 


\section{High Flow Through a Nasal Cannula and CPAP Effect in a Simulated Infant Model}

Table 1. Tidal Volume Results

\begin{tabular}{lc}
\hline \hline $\begin{array}{c}\text { Muscle Pressure } \\
\left(\mathrm{cm} \mathrm{H}_{2} \mathrm{O}\right)\end{array}$ & $\begin{array}{c}\text { Resultant Tidal Volume } \\
(\mathrm{mL})\end{array}$ \\
\hline 6.5 & 3 \\
13.0 & 6 \\
19.5 & 9 \\
26.0 & 12 \\
\hline
\end{tabular}

used the identical model that Cook and colleagues used to evaluate the effects of imposed resistance on $\mathrm{V}_{\mathrm{T}}$ with 5 neonatal CPAP systems. ${ }^{12}$ That prior work served as a model validation through a CPAP control group.

\section{Lung Simulator Settings}

An active lung model simulated a sick neonate of approximately $1 \mathrm{~kg}$. Lung compliance and resistance were set at $0.5 \mathrm{~mL} / \mathrm{cm} \mathrm{H}_{2} \mathrm{O}$ and $125 \mathrm{~cm} \mathrm{H}_{2} \mathrm{O} / \mathrm{L} / \mathrm{s}$, respectively. Muscle pressure $\left(\mathrm{P}_{\text {mus }}\right)$, which is the pressure generated by the respiratory muscles to expand the thoracic cage and the lungs, was simulated by setting a waveform and adjusting the amplitude on the lung simulator. The $\mathrm{P}_{\text {mus }}$ waveform was: increase $33 \%$, hold $0 \%$, and release $33 \%$. $\mathrm{P}_{\text {mus }}$ amplitude was adjusted to deliver a range of $\mathrm{V}_{\mathrm{T}}$ from $3 \mathrm{~mL}$ to $12 \mathrm{~mL}$ (Table 1).

\section{Procedure}

The respiratory rate on the active lung model was set at 65 breaths/min, and the inspiratory-expiratory ratio was held constant at 1:2 with each $\mathrm{V}_{\mathrm{T}}$ tested over the range of volumes $(3-12 \mathrm{~mL})$. The prongs of a standard nasal cannula or cannula designed and approved to deliver low-flow oxygen therapy to infants and children, were inserted into each nares size model. We tested 3 cannula sizes: premature infant (Comfort Flow model 2411-04, Hudson RCI, Durham, North Carolina), infant (Comfort Flow model 2411-03, Hudson RCI, Durham, North Carolina), and pediatric (Comfort Flow model 2411-02, Hudson RCI, Durham, North Carolina). Despite the labels "infant" and "pediatric," any of these 3 sizes might be used on a $1-\mathrm{kg}$ neonate, depending on the geometry of the infant's nares. Nasal cannula size selection was therefore based on the simulated nares opening sizes (small, medium, and large). As with the selection of a nasal cannula for clinical use in infants and children, the cannula size was chosen such that the nares were never occluded. The presence of leak was confirmed by calculating the ratio of the area of the cannula prong to the nares. Each cannula size was inserted into the nares opening and evaluated for the presence of occlusion. Table 2 shows the matrix of cannula size to nares opening, expressed as percent occlusion.
Table 2. Percent Occlusion of the Model Nares*

\begin{tabular}{lccc}
\hline \hline \multirow{2}{*}{ Cannula Size } & \multicolumn{3}{c}{ Nares Size } \\
\cline { 2 - 4 } & Small & Medium & Large \\
\hline Premature infant (model 2411-04) & 16 & 12 & 11 \\
Infant (model 2411-03) & 27 & 21 & 19 \\
Pediatric (model 2411-02) & 37 & 30 & 27 \\
& & & \\
* Values are percent occlusion, calculated as the ratio of the nares opening area to the nasal \\
cannula prong area.
\end{tabular}

Baseline $\mathrm{V}_{\mathrm{T}}$ values were obtained with the nasal cannula inserted into the nares model without flow. Cannula flow rates were then adjusted from $2-6 \mathrm{~L} / \mathrm{min}$, in $1-\mathrm{L} / \mathrm{min}$ increments, with each $\mathrm{V}_{\mathrm{T}}$ tested.

\section{Data Collection and Analysis}

The lung simulator's software was used to average data over 20 breaths for each experimental condition. Breath start volume (ie, breath detection) threshold was set at $0.1 \mathrm{~mL}$. Outcome variables were highest and lowest airway pressure, mean airway pressure, and $\mathrm{V}_{\mathrm{T}}$ drop due to loading effects, defined as baseline $\mathrm{V}_{\mathrm{T}}$ of lung simulator not connected to the nasal prongs minus the $\mathrm{V}_{\mathrm{T}}$ with nasal prongs in place. Screen captures from the lung simulator were also obtained of airway pressure and volume at the different flow rates. Data were entered into SPSS 17.0 (SPSS, Chicago, Illinois) for analysis.

\section{Results}

$\mathrm{V}_{\mathrm{T}}$ decreased as flow increased over the range of flows with each cannula size at each $\mathrm{P}_{\text {mus }}$ (Fig. 3). The changes in the baseline $\mathrm{V}_{\mathrm{T}}$ delivered by the lung simulator and the $\mathrm{V}_{\mathrm{T}}$ recorded with nasal prongs in place at baseline with zero flow and across the range of flows were statistically significant for each of the cannula sizes (Table 3). The peak pressure changes ranged from less than $0.2 \mathrm{~cm} \mathrm{H}_{2} \mathrm{O}$ to approximately $3.5 \mathrm{~cm} \mathrm{H}_{2} \mathrm{O}$ across all models and experimental conditions. Flows of $\geq 2 \mathrm{~L} /$ min generated positive expiratory pressure with all cannula sizes in all experimental conditions. The expiratory pressure range was $0.04-1.3 \mathrm{~cm} \mathrm{H}_{2} \mathrm{O}$.

Figure 4 shows the baseline, peak, mean, and PEEP changes over the range of flows used. Figure 5 shows the airway pressures observed during inspiration at the highest and lowest $\mathrm{P}_{\text {mus }}$ values, as well as the pressure drop recorded for each cannula size during the initiation of a spontaneous breath for each experimental condition. Figure 6 shows representative airway pressure, volume, and $\mathrm{P}_{\text {mus }}$ waveforms. 

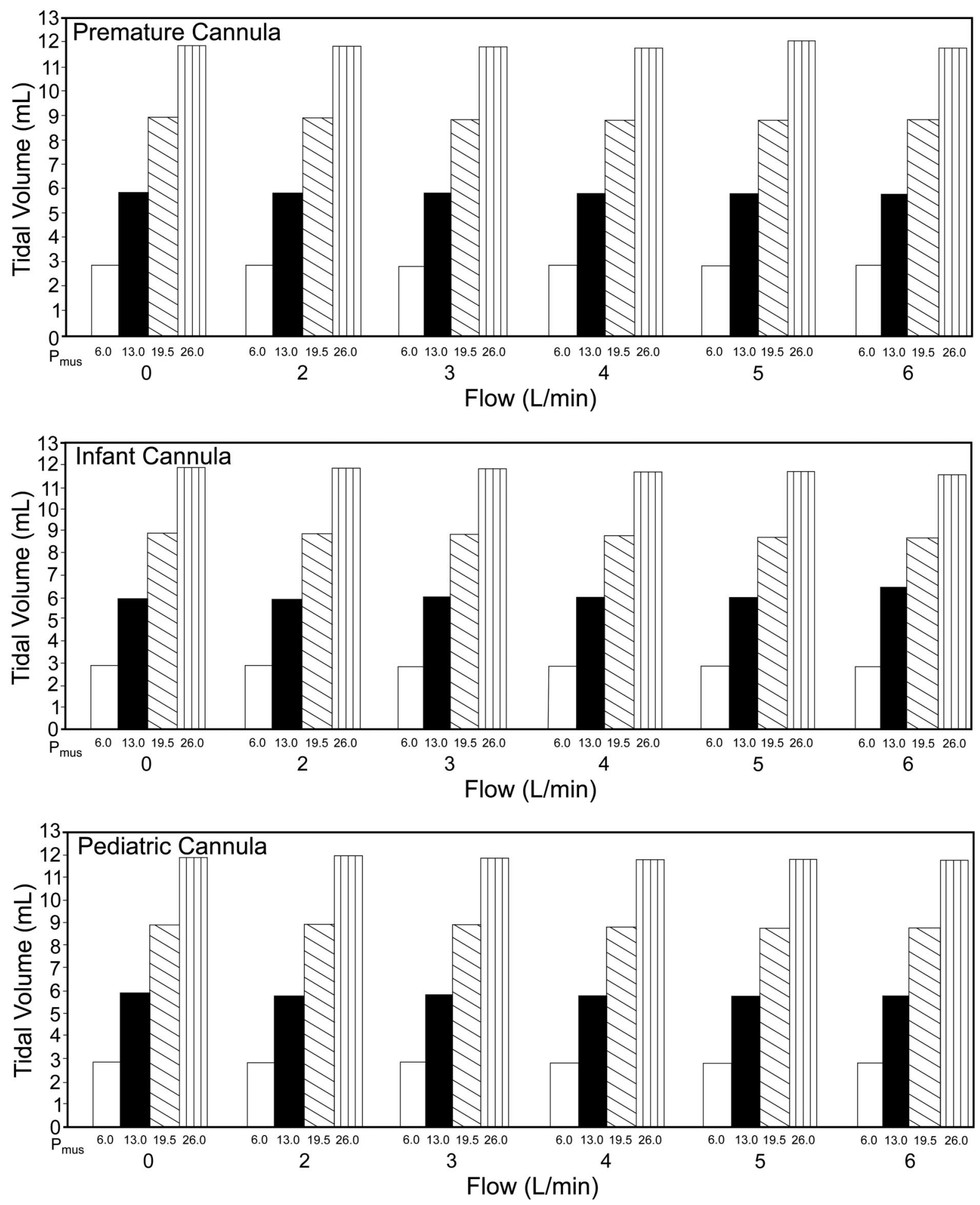

Fig. 3. Changes in tidal volume at 6 flows $(0-6 \mathrm{~L} / \mathrm{min})$ and 4 simulated muscle pressure $\left(P_{\text {mus }}\right)$ settings.

\section{Discussion}

Many studies have described the therapeutic value of CPAP. The literature reports that early use of CPAP may reduce the need for intubation, ${ }^{13-15}$ facilitate successful extubation, ${ }^{16,17}$ and reduce the incidence of chronic lung disease. ${ }^{18-20}$ The therapeutic effectiveness of CPAP is in part dependent on the ability to achieve and maintain a 


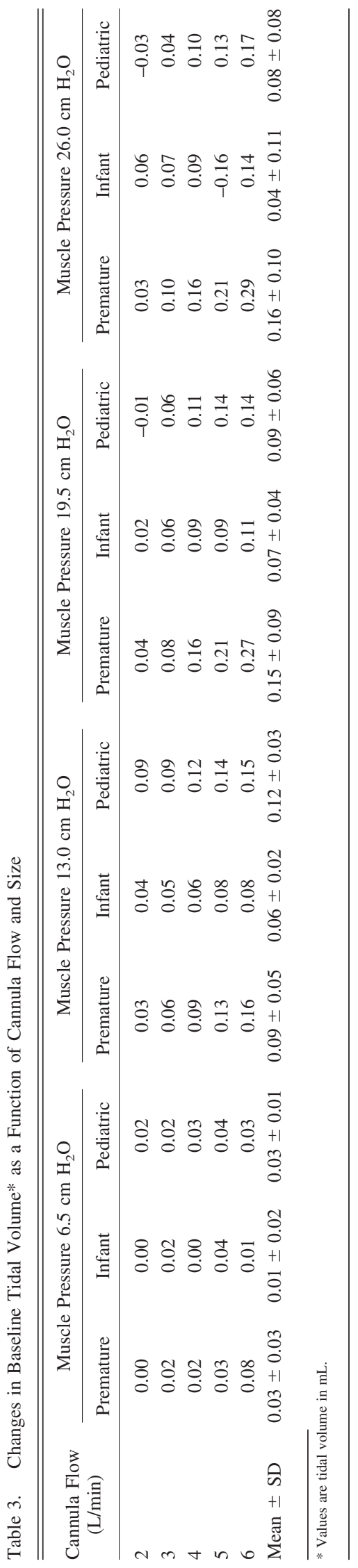

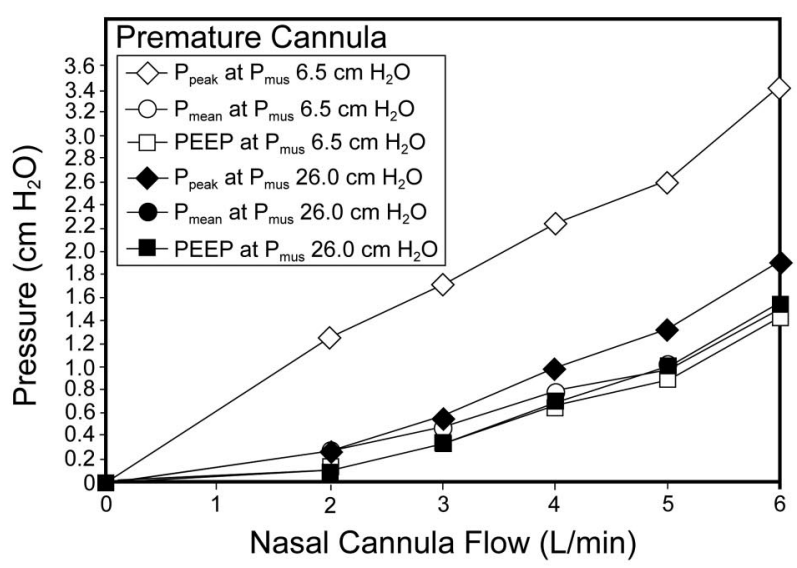
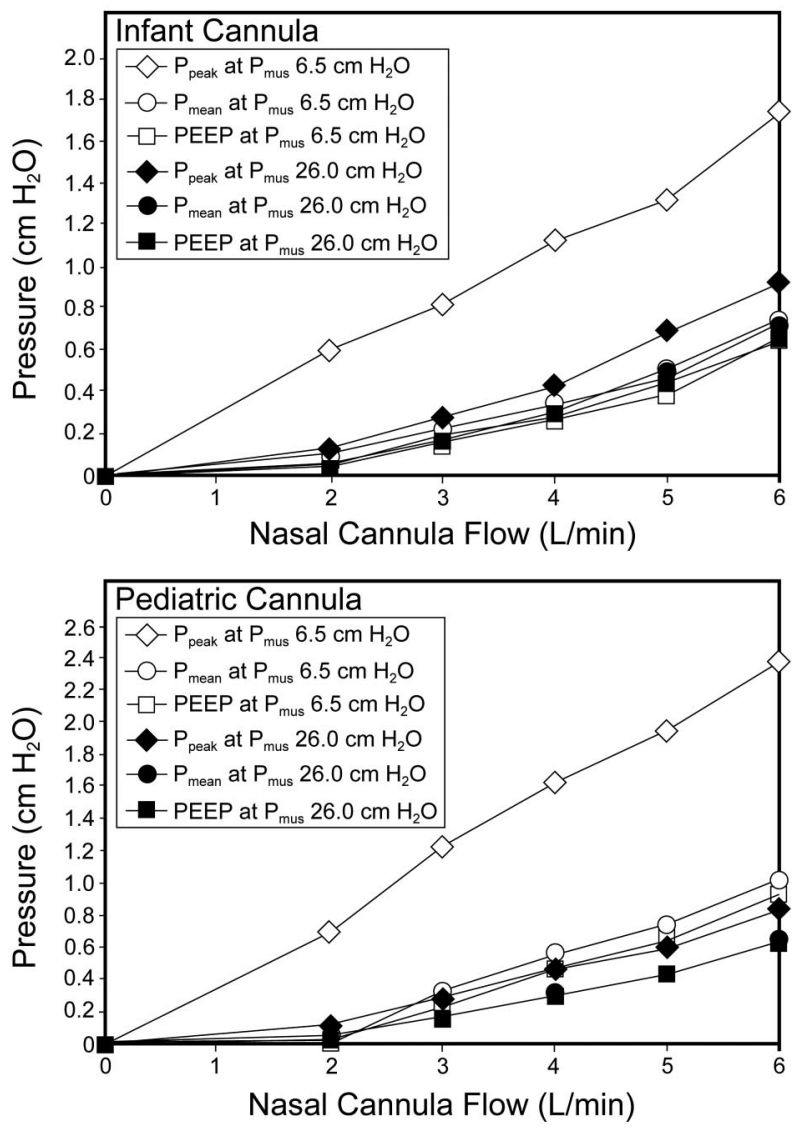

Fig. 4. Changes in peak pressure, mean pressure, and PEEP at 6 flows $(0-6 \mathrm{~L} / \mathrm{min})$ and 2 simulated muscle pressure $\left(P_{\text {mus }}\right)$ settings.

constant distending pressure. A variety of delivery systems and interfaces can be used to deliver CPAP, ranging from a flow meter connected to a traditional nasal cannula to a sophisticated mechanical ventilator. Therefore it is essential for the clinician to have a thorough understanding of how the CPAP delivery system works and a working knowledge of its limitations.

There have been a few reports of success using highflow nasal cannula, which mirror the results of CPAP. In 

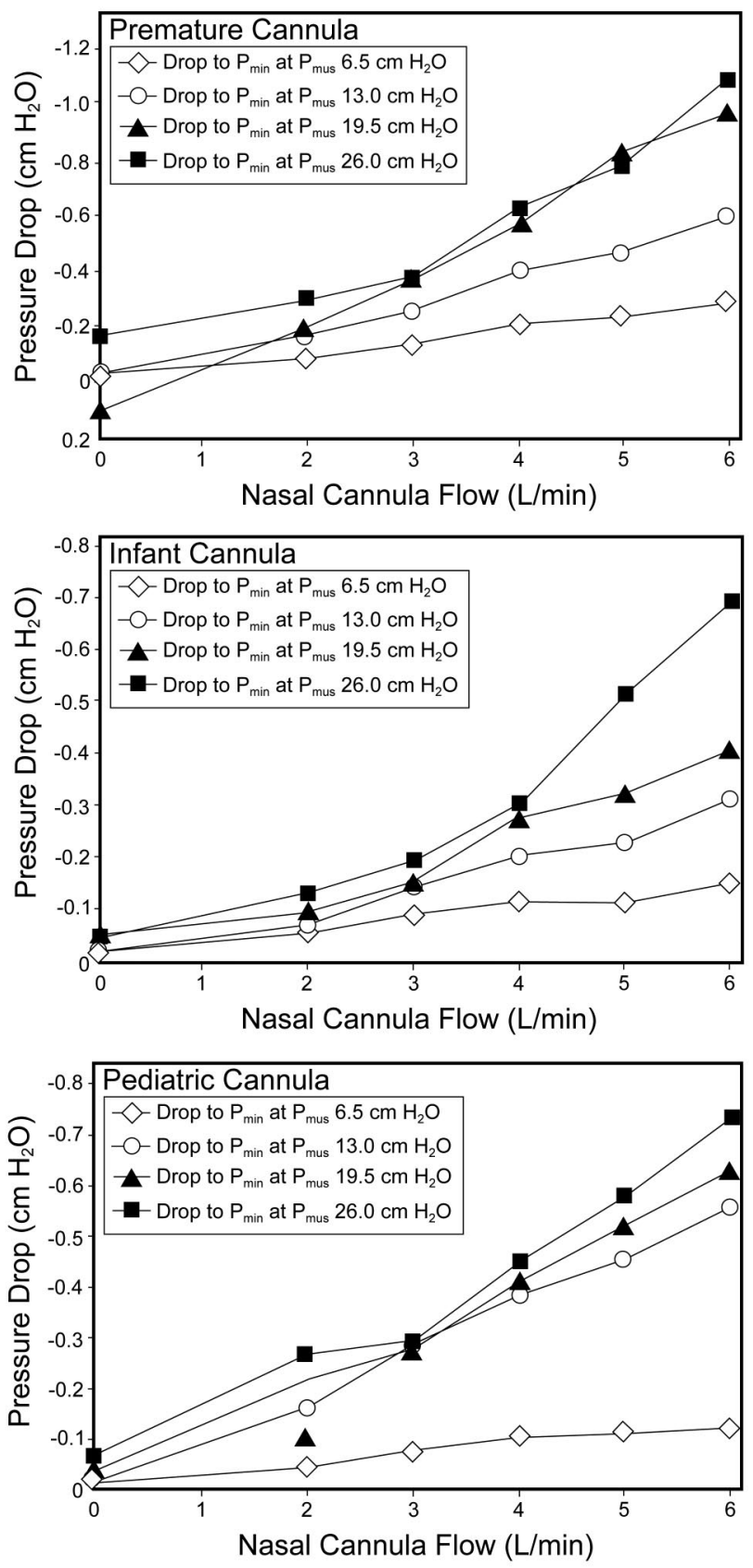

Fig. 5. Pressure drop $\left(P_{\min }\right)$ at 6 flows $(0-6 \mathrm{~L} / \mathrm{min})$ and 4 simulated muscle pressure $\left(P_{\text {mus }}\right)$ settings during the initiation of an inspiratory effort.

a review of the literature, Dani et al mention only 3 published studies of the CPAP effect with a traditional nasal cannula set at "high" flow (ie, $>2 \mathrm{~L} / \mathrm{min}$ ). ${ }^{21}$ The amount of positive expiratory pressure generated depended on several factors, including the inner diameter of the nasal cannula prongs, the gas flow through the cannula, and the anatomy of the infant's airway. ${ }^{17}$ PEEP was also generated at the airway opening of our simulated model. Consistent with the findings reported in the literature, the distending pressure varied across the range for flows for each of the models tested. The pressure changes obtained in our simulated model were less than those used intentionally with CPAP devices, and the maximum pressure was less than $2 \mathrm{~cm} \mathrm{H}_{2} \mathrm{O}$, which is probably not clinically important. Our findings were comparable to the measured changes in continuous distending pressure found by Locke and colleagues with a $0.2-\mathrm{cm}$ nasal cannula at high flow. ${ }^{22}$ The generation of appreciable CPAP effect across the range of flows $(0.5-2.0 \mathrm{~L} / \mathrm{min})$ tested by Locke and colleagues occurred with a $0.3-\mathrm{cm}$ cannula. In preterm infants, larger nasal prongs enabled greater mean pressure: specifically, $9.8 \mathrm{~cm} \mathrm{H}_{2} \mathrm{O} .{ }^{18}$

The effect of airway pressures on $\mathrm{V}_{\mathrm{T}}$ can be appreciated by considering the theoretical expectation from the equation of motion for the respiratory system:

$\mathrm{P}_{\text {airway }}+\mathrm{P}_{\text {mus }}=$ elastance $\times$ volume + resistance $\times$ flow

This equation shows that if airway pressure $\left(\mathrm{P}_{\text {airway }}\right)$ drops and $\mathrm{P}_{\text {mus }}$ stays constant, then volume and flow must drop. Our data show a small drop in airway pressure during inspiration, because the high-flow nasal cannula system is a poor CPAP generator. Thus, the small drop in $\mathrm{V}_{\mathrm{T}}$ relative to zero flow is expected. We found clinically unimportant $\mathrm{V}_{\mathrm{T}}$ changes with each of the tested cannula sizes, across the range of flows.

\section{Limitations}

Perhaps the most obvious limitation of this study was that we could not account for the effect of the pressure and/or volume changes that might occur with infants as a result of variable leak secondary to the opening and closing of the infant's mouth. In an observational study of 27 pre-term to term infants (gestational age 25-40 weeks), Kubicka et al investigated the level of delivered continuous positive airway pressure by measuring oral cavity pressure with the mouth closed in infants of various weights and ages treated with heated humidified high-flow nasal cannula, at flows of 1-5 L/min. At the tested flows (1$5 \mathrm{~L} / \mathrm{min}$ ), nasal cannula did not deliver positive airway pressure when the infant's mouth was open. ${ }^{23}$ Clinically important, and unpredictable levels of positive expiratory pressure were achieved only in the smallest infants and the highest flows, with the mouth fully closed. ${ }^{19}$ This in vivo experiment was also limited by the inability to duplicate the variable occlusion of the nares and intranasal airway by secretions or welling, for example in this experimental model. 

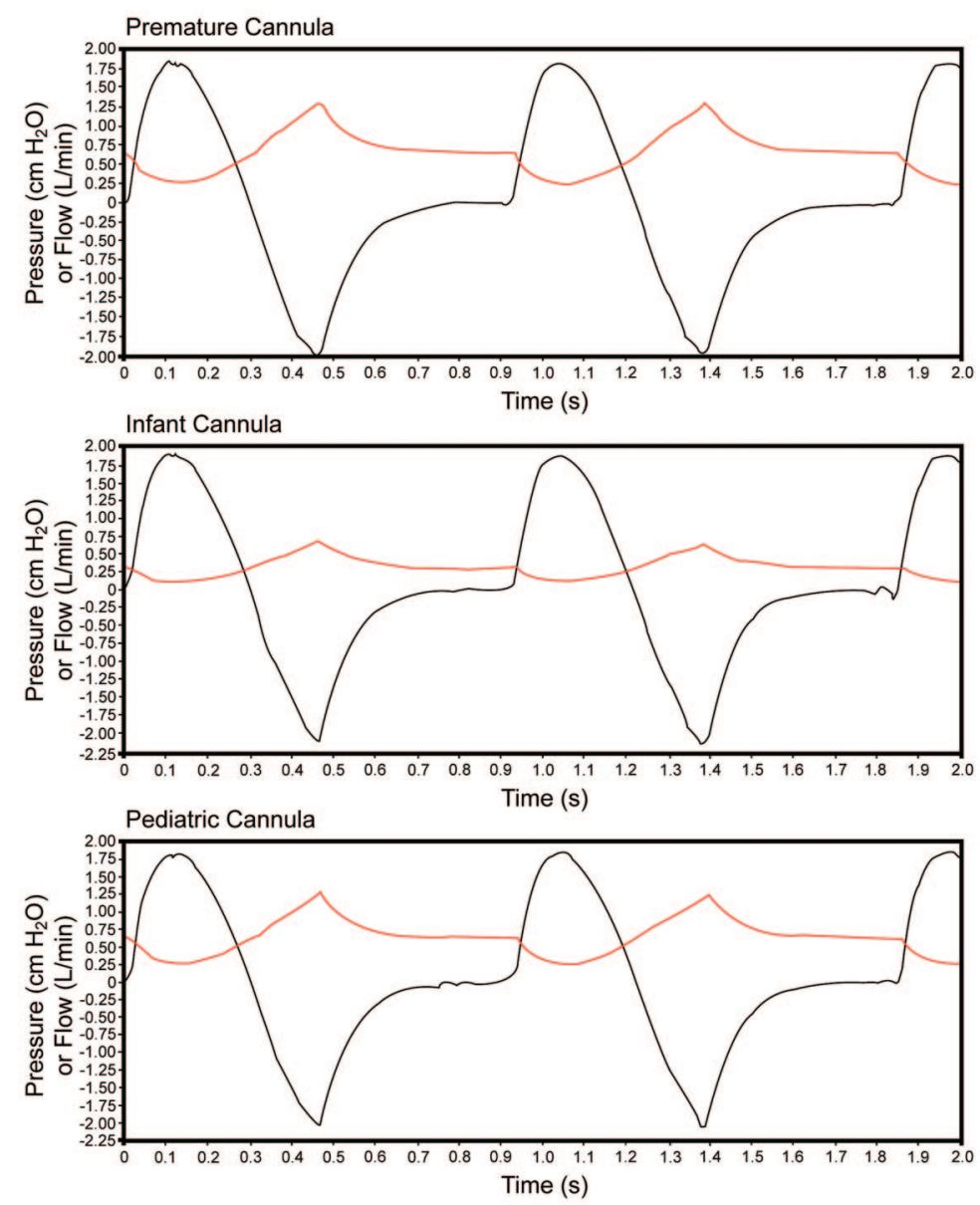

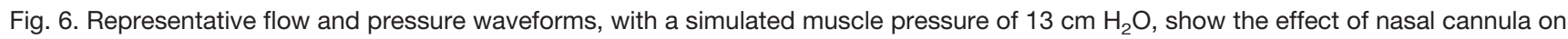
airway pressure (red waveforms) and flow (black waveforms, scale $\times 0.1$ ).

The convenience of using conventional nasal cannula may enhance the ability of the neonate to participate in activities such as kangarooing and oral feeding, both of which have notable impacts on patient outcomes. As research continues on high-flow nasal cannula we must determine the most appropriate place to measure airway pressure as well as its potential effects on the neonate. Additionally, we need to find consensus regarding patient selection, cannula selection, and flow selection to achieve practice guidelines. Since our findings cannot be extrapolated to the application of high flows using other nasal flow delivery devices, additional research on the CPAP effect with available high-flow nasal cannulas may be of interest.

\section{Conclusions}

In our model with several nasal cannulas correctly sized for several nares sizes, high flow did not generate clinically important continuous airway pressure. The decreases in spontaneous $\mathrm{V}_{\mathrm{T}}$ due to loading effects for each of the models across the range of flows were also negligible.

\section{REFERENCES}

1. Pandit PB, Courtney SE, Pyon KH, Saslow JG, Habib RH. Work of breathing during constant- and variable-flow nasal continuous positive airway pressure in preterm neonates. Pediatrics 2001;108(3): 682-685.

2. Stewart AR, Finer NN, Peters KL. Effects of alterations of inspiratory and expiratory pressures and inspiratory/expiratory ratios on mean airway pressure, blood gases, and intracranial pressure. Pediatrics 1981;67(4):474-481.

3. Speidel BD, Dunn PM. Effect of continuous positive airway pressure on breathing pattern of infants with respiratory-distress syndrome. Lancet 1975;1(7902):302-304.

4. Miller MJ, Carlo WA, Martin RJ. Continuous positive airway pressure selectively reduces obstructive apnea in preterm infants. J Pediatr 1985;106(1):91-94.

5. Krouskop RW, Brown EG, Sweet AY. The early use of continuous positive airway pressure in the treatment of idiopathic respiratory distress syndrome. J Pediatr 1975;87:263-267. 


\section{High Flow Through a Nasal Cannula and CPAP Effect in a Simulated Infant Model}

6. Annibale DJ, Hulsey TC, Engstrom PC, Wallin LA, Ohning BL. Randomized, controlled trial of nasopharyngeal continuous positive airway pressure in the extubation of very low birth weight infants. J Pediatr 1994;124(3):455-460.

7. Barrington KJ. Management of respiratory failure in the preterm infant. Minerva Pediatr 2008;60(2):183-192.

8. Lee KS, Dunn MS, Fenwick M, Shennan AT. A comparison of underwater bubble continuous positive airway pressure with ventilator-derived continuous positive airway pressure in premature neonates ready for extubation. Biol Neonate 1998;73(2):69-75.

9. Sankar MJ, Sankar J, Agarwal R, Paul VK, Deorari AK. Protocol for administering continuous positive airway pressure in neonates. Indian J Pediatr 2008;75(5):471-478.

10. Kopelman AE, Holbert D. Use of oxygen cannulas in extremely low birthweight infants is associated with mucosal trauma and bleeding, and possibly with coagulase-negative staphylococcal sepsis. J Perinatol 2003;23(2):94-97.

11. Kopelman AE. Airway obstruction in two extremely low birthweight infants treated with oxygen cannulas. J Perinatol 2003;23(2):164-165.

12. Cook SE, Fedor KL, Chatburn RL. Effects of imposed resistance on tidal volume with 5 neonatal nasal continuous positive airway pressure systems. Respir Care 2010;55(5):544-548.

13. Gittermann MK, Fusch C, Gittermann AR, Regazzoni BM, Moessinger AC. Early nasal continuous positive airway pressure treatment reduces the need for intubation in very low birth weight infants. Eur J Pediatr 1997;156(5):384-388.

14. Kamper J, Wulff K, Larsen C, Lindequist S. Early treatment with nasal continuous positive airway pressure in very low-birth-weight infants. Acta Paediatr Scand 1993;82(2):193-197.
15. Lundstrom KE, Griesen G. Early treatment with nasal-CPAP. Acta Paediatr Scand 1993;82(10):856.

16. Gupta A, Sunha SK, Tin W, Donn SM. A randomized controlled trial of post-extubation bubble continuous positive airway pressure versus Infant Flow Driver continuous positive airway pressure in preterm infants with respiratory distress syndrome. J Pediatr 2009;154(5): 645-650.

17. Moretti C, Gizzi C, Papoff P, Lampariello S, Capoferri M, Calcagnini G, Bucci G. Comparing the effects of nasal synchronized intermittent positive pressure ventilation (nSIPPV) and nasal continuous positive airway pressure (nCPAP) after extubation in very low birth weight infants. Early Hum Dev 1999;56(2-3):167-177.

18. Poets CF, Sens B. Changes in intubation rates and outcome of very low birth weight infants: a population-based study. Pediatrics 1996; 98(1):24-27.

19. Kamper J, Ringsted C. Early treatment of idiopathic respiratory distress syndrome using binasal continuous positive airway pressure. Acta Paediatr Scand 1990;79(6-7):581-586.

20. Patel D, Greenough A. Does nasal CPAP reduce bronchopulmonary dysplasia (BPD)? Acta Paediatr 2008;97(10):1314-1317.

21. Dani C, Pratesi S, Migliori C, Bertini G. High flow nasal cannula therapy as respiratory support in the preterm infant. Pediatric Pulmonology 2009;44(7):629-634.

22. Locke RG, Wolfson MR, Shaffer TH, Rubenstein D, Greenspan JS Inadvertent administration of positive end-expiratory pressure during nasal cannula flow. Pediatrics 1993;91(1):135-138.

23. Kubicka ZJ, Limauro J, Darnall RA. Heated, humidified high flow nasal cannula therapy: yet another way to deliver continuous positive airway pressure? Pediatrics 2008;121(1):82-88.

This article is approved for Continuing Respiratory Care Education credit. For information and to obtain your CRCE

(free to AARC members) visit 